Article

\title{
Iron Status and Cancer Risk in UK Biobank: A Two-Sample Mendelian Randomization Study
}

\author{
Shuai Yuan 1,2®, Paul Carter ${ }^{3}$, Mathew Vithayathil ${ }^{4}$, Siddhartha Kar ${ }^{3,5}$, \\ Edward Giovannucci ${ }^{6,7}$, Amy M. Mason ${ }^{3}{ }^{-}$, Stephen Burgess ${ }^{3,8}$ and Susanna C. Larsson 1,2,* \\ 1 Unit of Cardiovascular and Nutritional Epidemiology, Institute of Environmental Medicine, Karolinska \\ Institutet, SE-171 77 Stockholm, Sweden; shuai.yuan@ki.se \\ 2 Department of Surgical Sciences, Uppsala University, SE-751 85 Uppsala, Sweden \\ 3 Department of Public Health and Primary Care, University of Cambridge, Cambridge CB1 8RN, UK; \\ paul_richard_carter@outlook.com (P.C.); siddhartha.kar@bristol.ac.uk (S.K.); \\ am2609@medschl.cam.ac.uk (A.M.M.); sb452@medschl.cam.ac.uk (S.B.) \\ 4 MRC Cancer Unit, University of Cambridge, Cambridge CB2 0XZ, UK; mat2k89@gmail.com \\ 5 MRC Integrative Epidemiology Unit, Bristol Medical School, University of Bristol, Bristol BS8 1QU, UK \\ 6 Departments of Epidemiology and Nutrition, Harvard T. H. Chan School of Public Health, Boston, \\ MA 02115, USA; egiovann@hsph.harvard.edu \\ 7 Channing Division of Network Medicine, Department of Medicine, Brigham and Women's Hospital, and \\ Harvard Medical School, Boston, MA 02115, USA \\ 8 MRC Biostatistics Unit, University of Cambridge, Cambridge CB2 0SR, UK \\ * Correspondence: susanna.larsson@ki.se; Tel.: +46-8-52486059
}

Received: 22 January 2020; Accepted: 18 February 2020; Published: 19 February 2020

\begin{abstract}
We conducted a two-sample Mendelian randomization study to explore the associations of iron status with overall cancer and 22 site-specific cancers. Single-nucleotide polymorphisms for iron status were obtained from a genome-wide association study of 48,972 European-descent individuals. Summary-level data for breast and other cancers were obtained from the Breast Cancer Association Consortium and UK Biobank. Genetically predicted iron status was positively associated with liver cancer and inversely associated with brain cancer but not associated with overall cancer or the other 20 studied cancer sites at $p<0.05$. The odds ratios of liver cancer were $2.45(95 \% \mathrm{CI}, 0.81$, $7.45 ; p=0.11), 2.11(1.16,3.83 ; p=0.02), 10.89(2.44,48.59 ; p=0.002)$ and $0.30\left(0.17,0.53 ; p=2 \times 10^{-5}\right)$ for one standard deviation increment of serum iron, transferrin saturation, ferritin and transferrin levels, respectively. For brain cancer, the corresponding odds ratios were $0.69(0.48,1.00 ; p=0.05)$, $0.75(0.59,0.97 ; p=0.03), 0.41(0.20,0.88 ; p=0.02)$ and $1.49(1.04,2.14 ; p=0.03)$. Genetically high iron status was positively associated with liver cancer and inversely associated with brain cancer.
\end{abstract}

Keywords: iron; transferrin saturation; ferritin; serum transferrin; cancer; Mendelian randomization

\section{Introduction}

Hereditary hemochromatosis, an autosomal recessive disorder characterized by a progressive iron overload, greatly increases the risk of developing hepatocellular carcinoma and non-neoplastic liver diseases [1,2]. However, the carcinogenic effects of physiologically high levels of iron, an essential nutrient influencing cell proliferation and growth [3], on liver cancer and non-hepatic malignancies are unknown.

A randomized trial of 1277 individuals with peripheral arterial disease (636 in iron reduction group through venesection and 641 in control group) found that in the iron reduction group, who received regular phlebotomy, the risk of overall cancer, cancer-specific mortality and all-cause mortality was lower than in the control group after 4.5 years of follow-up [4]. However, findings of observational 
studies are inconsistent or scarce concerning the effects of iron status on individual cancers, such as colorectal, breast and oesophagal cancer [5-11]. Consequently, whether iron status (which is routinely measured in clinical practice as serum iron, transferrin saturation, ferritin and transferrin) plays a role in the development of site-specific cancer remains inconclusive.

Mendelian randomization (MR) is an approach that can strengthen the inference about the causal nature of exposure-outcome associations by exploiting genetic variants as instrumental variables of the exposures [12]. This method has the strength of minimizing confounding as genetic variants are randomly assorted at conception, thereby being irrelevant to self-selected lifestyle and environmental factors. Additionally, it overcomes reverse causality since allelic randomization antedates the disease's development. We conducted a two-sample MR study to systematically assess the possible causal associations of four iron status biomarkers with risk of overall and 22 site-specific cancers.

\section{Methods}

\subsection{Study Design Overview}

We used a two-sample MR approach by using summary statistics data from a genome-wide association study (GWAS) of iron status, the Breast Cancer Association Consortium and the UK Biobank, which obtained appropriate patient consent and ethical approval. Overall cancer was treated as the primary outcome and the site-specific cancers as secondary outcomes. Information on the studies and consortia used in the present study is summarized in Supplementary Table S1. The present study was approved by the Swedish Ethical Review Authority.

\subsection{Instrumental Variable Selection}

In a GWAS consisting of 48,972 individuals of European ancestry, the Genetics of Iron Status Consortium identified five single nucleotide polymorphisms (SNP) associated with serum iron and transferrin saturation, six SNPs associated with ferritin and eight SNPs associated with transferrin at the genome-wide significance threshold $\left(p<5 \times 10^{-8}\right)$ [13] (Table 1). Among those SNPs, three SNPs (rs1800562 and rs1799945 in HFE and rs855791 in TMPRSS6) showed a robust and consistent association with a systemic iron status and explained the majority of variance for each iron status biomarker [14] and have been used as instrumental variables for iron status in previous MR studies [14-16]. The SNPs were uncorrelated $\left(\mathrm{R}^{2}<0.01\right)$ and explained $3.4 \%, 7.2 \%, 6.9 \%$ and $0.9 \%$ of the variance for serum iron, transferrin, transferrin saturation and ferritin levels, respectively [13]. Details of the SNPs associated with the iron status biomarkers are presented in Table 1.

\subsection{Outcome Data Sources}

Summary-level data for breast cancer (including estrogen receptor positive and negative breast cancer) were obtained from the Breast Cancer Association Consortium with 228,951 individuals of European ancestry (122,977 cancer cases and 105,974 controls) [17]. The GWAS for breast cancer used 1000 Genomes Project (Phase 3) reference panel in imputation stage and the logistic regression analyses adjusted for genetic principal components and country.

Summary-level data for overall cancer and 22 site-specific cancers were extracted from the UK Biobank [18]. This cohort study recruited around 500,000 adults, aged 40 to 69 years, across the UK from 2006 to 2010. To reduce population stratification bias, we confided the study population to European-descent individuals. After exclusion of related individuals (third-degree relatives or closer), low call rate, and excess heterozygosity (3 or more standard deviations from the mean), 367,643 participants remained in the analyses and were followed up until March 31, 2017, or death. In total, there were 75,037 cancer cases at any site. 
Table 1. SNPs associated with iron status biomarkers at genome-wide significance level and included in the main analyses with three primary SNPs and secondary analyses of all SNPs.

\begin{tabular}{|c|c|c|c|c|c|c|c|c|c|c|c|c|c|c|c|}
\hline \multirow{2}{*}{ SNP } & \multirow{2}{*}{$\begin{array}{c}\text { Nearby } \\
\text { Gene }\end{array}$} & \multirow{2}{*}{ EA } & \multirow{2}{*}{ EAF } & \multicolumn{3}{|c|}{ Serum Iron, $\mu \mathrm{mol} / \mathrm{L}$} & \multicolumn{3}{|c|}{ Transferrin Saturation, $\%$} & \multicolumn{3}{|c|}{$\log 10$ Ferritin, $\mu \mathrm{g} / \mathrm{L}$} & \multicolumn{3}{|c|}{ Ferritin, $\mathrm{g} / \mathrm{L}$} \\
\hline & & & & Beta & SE & $p$ & Beta & SE & $p$ & Beta & SE & $p$ & Beta & SE & $p$ \\
\hline rs $1800562 *$ & HFE & A & 0.07 & 0.328 & 0.016 & $2.9 \times 10^{-97}$ & 0.577 & 0.016 & $2.2 \times 10^{-270}$ & 0.204 & 0.016 & $1.5 \times 10^{-38}$ & -0.479 & 0.016 & $8.9 \times 10^{-196}$ \\
\hline rs1799945* & HFE & G & 0.15 & 0.189 & 0.010 & $1.1 \times 10^{-81}$ & 0.231 & 0.010 & $5.1 \times 10^{-109}$ & 0.065 & 0.010 & $1.7 \times 10^{-10}$ & -0.114 & 0.010 & $9.4 \times 10^{-30}$ \\
\hline rs855791* & TMPRSS6 & G & 0.55 & 0.181 & 0.007 & $4.3 \times 10^{-139}$ & 0.190 & 0.007 & $6.4 \times 10^{-137}$ & 0.055 & 0.007 & $1.4 \times 10^{-14}$ & -0.044 & 0.007 & $2.0 \times 10^{-9}$ \\
\hline rs8177240 & TF & G & 0.35 & 0.066 & 0.007 & $6.6 \times 10^{-20}$ & 0.100 & 0.008 & $7.2 \times 10^{-38}$ & & & & 0.380 & 0.007 & $8.4 \times 10^{-610}$ \\
\hline rs7385804 & TFR2 & $\mathrm{A}$ & 0.62 & 0.064 & 0.007 & $1.4 \times 10^{-18}$ & 0.054 & 0.008 & $6.1 \times 10^{-12}$ & & & & & & \\
\hline rs744653 & AC013439.4 & $\mathrm{C}$ & 0.16 & & & & & & & 0.089 & 0.010 & $8.4 \times 10^{-19}$ & & & \\
\hline rs411988 & TEX14 & G & 0.44 & & & & & & & 0.044 & 0.007 & $1.6 \times 10^{-10}$ & & & \\
\hline rs651007 & $A B O$ & $\mathrm{C}$ & 0.79 & & & & & & & 0.050 & 0.009 & $1.3 \times 10^{-8}$ & & & \\
\hline rs4921915 & NAT2 & $\mathrm{A}$ & 0.76 & & & & & & & & & & 0.079 & 0.009 & $7.1 \times 10^{-19}$ \\
\hline rs174577 & FADS2 & $\mathrm{A}$ & 0.36 & & & & & & & & & & 0.062 & 0.007 & $2.3 \times 10^{-17}$ \\
\hline rs9990333 & TFRC & $\mathrm{C}$ & 0.53 & & & & & & & & & & 0.051 & 0.007 & $2.0 \times 10^{-13}$ \\
\hline rs6486121 & ARNTL & $\mathrm{C}$ & 0.34 & & & & & & & & & & 0.046 & 0.007 & $3.9 \times 10^{-10}$ \\
\hline
\end{tabular}

EA indicates effect allele; EAF, effect allele frequency; SE, standard error; SNP, single nucleotide polymorphism. * Three SNPs were included in the main analyses. 


\subsection{Statistical Analysis}

The inverse-variance weighted method with random-effects was used to assess the causal associations between iron status and cancer risk. In the main analysis, we employed three genetic variants (rs1800562 and rs1799945 in HFE and rs855791 in TMPRSS6), explaining the major proportion of variance for iron status, as instrumental variables. All SNPs reaching the genome-wide significance level for individual iron status indicators were used in the sensitivity analysis. Furthermore, we performed sensitivity analyses, including the weighted median [19] and MR-Egger methods [20], for associations with $p<0.05$ in the inverse-variance weighted models. Odds ratios (ORs) of cancer are per one standard deviation (SD) increase in genetically predicted serum iron, log10 ferritin, ferritin saturation and transferrin levels in all analyses. Power calculations were based on a method designed for a binary outcome [21]; results from these analyses are displayed in Supplementary Figure S1. All statistical analyses were two-sided and performed in Stata/SE 15.0 and R 3.6.0 software. We searched the PhenoScanner V2 database [22] (a database of human genotype-phenotype associations) to detect possible pleiotropy for the SNPs associated with the iron status biomarkers. The $p$ values were not strictly used to define statistical significance, but we interpreted the results based on the strengths of the associations [23] as well as the consistency across sensitivity analyses.

\section{Results}

Genetically predicted high iron status was positively associated with liver cancer and inversely associated with brain cancer but was not associated with overall cancer and the other 20 cancers (Table 2). The OR of liver cancer was 2.45 (95\% CI, 0.81, 7.45; $p=0.11), 2.11(95 \% \mathrm{CI}, 1.16,3.83 ; p=0.02)$, $10.89(95 \% \mathrm{CI}, 2.44,48.59 ; p=0.002)$ and $0.30\left(95 \% \mathrm{CI}, 0.17,0.53 ; p=2 \times 10^{-5}\right)$ for one SD increase in genetically predicted serum iron, $\log 10$ ferritin, ferritin saturation and transferrin levels. Results were consistent across sensitivity analyses (Table 3). Substantial heterogeneity among estimates of individual SNPs $\left(\mathrm{I}^{2}=76, p=0.01\right)$ and potential pleiotropy (intercept -0.662 (95\% CI -1.124, -0.199); $p=5.00 \times 10^{-3}$ ) were detected in the analysis of serum iron levels. The associations of iron status biomarkers with liver cancer were mainly driven by rs1800562 in HFE (Table 3), which had the largest effect on iron status (Table 1). Even though none of the associations remained significant, the magnitude and direction of associations were similar after excluding that SNP (Supplementary Table S2). The OR of brain cancer was 0.69 (95\% CI, 0.48, 1.00; $p=0.05), 0.75$ (95\% CI, 0.59, 0.97; $p=0.03), 0.41(95 \%$ CI, 0.20, 0.88; $p=0.02)$ and $1.49(95 \% \mathrm{CI}, 1.04,2.14 ; p=0.03)$ per one SD increment of serum iron, $\log 10$ ferritin, ferritin saturation and transferrin levels. The results for brain cancer were consistent and robust in all sensitivity analyses, and no heterogeneity and pleiotropy was observed (Table 4). However, as for liver cancer, the associations did not remain after removing rs1800562 (Supplementary Table S2). 
Table 2. Associations between genetically predicted iron status, based on three primary SNPs, and overall cancer and 22 site-specific cancers.

\begin{tabular}{|c|c|c|c|c|c|c|c|c|c|}
\hline \multirow{2}{*}{ Cancer } & \multirow{2}{*}{ Cases } & \multicolumn{2}{|c|}{ Serum Iron } & \multicolumn{2}{|c|}{ Transferrin Saturation } & \multicolumn{2}{|c|}{ Ferritin } & \multicolumn{2}{|c|}{ Transferrin } \\
\hline & & OR $(95 \% \mathrm{CI})$ & $p$ & OR $(95 \% \mathrm{CI})$ & $p$ & OR $(95 \% \mathrm{CI})$ & $p$ & OR $(95 \% \mathrm{CI})$ & $p$ \\
\hline Overall cancer (UKBB) & 75,037 & $1.03(0.96,1.10)$ & 0.47 & $1.01(0.96,1.07)$ & 0.65 & $1.03(0.88,1.21)$ & 0.71 & $1.00(0.92,1.08)$ & 0.99 \\
\hline \multicolumn{10}{|l|}{ Brain and head and neck } \\
\hline Brain & 810 & $0.69(0.48,1.00)$ & 0.05 & $0.75(0.59,0.97)$ & 0.03 & $0.41(0.20,0.88)$ & 0.02 & $1.49(1.04,2.14)$ & 0.03 \\
\hline Head and neck & 1615 & $0.93(0.71,1.23)$ & 0.63 & $0.97(0.79,1.19)$ & 0.77 & $0.93(0.50,1.72)$ & 0.81 & $0.99(0.73,1.34)$ & 0.95 \\
\hline \multicolumn{10}{|l|}{ Gastrointestinal tract } \\
\hline Oesophagus & 843 & $0.95(0.68,1.33)$ & 0.76 & $0.95(0.75,1.20)$ & 0.66 & $0.82(0.40,1.71)$ & 0.60 & $1.11(0.78,1.57)$ & 0.56 \\
\hline Stomach & 736 & $1.11(0.67,1.83)$ & 0.70 & $1.00(0.75,1.35)$ & 0.97 & $1.00(0.41,2.46)$ & 0.99 & $1.06(0.69,1.61)$ & 0.80 \\
\hline Colorectum & 5486 & $0.99(0.70,1.41)$ & 0.95 & $0.98(0.76,1.26)$ & 0.89 & $0.95(0.44,2.04)$ & 0.90 & $1.06(0.74,1.51)$ & 0.76 \\
\hline Pancreas & 1264 & $1.03(0.72,1.48)$ & 0.86 & $1.04(0.81,1.34)$ & 0.76 & $1.17(0.55,2.52)$ & 0.68 & $0.92(0.64,1.32)$ & 0.65 \\
\hline Liver & 324 & $2.45(0.81,7.45)$ & 0.11 & $2.11(1.16,3.83)$ & 0.01 & $10.89(2.44,48.59)$ & $2.0 \times 10^{-3}$ & $0.30(0.17,0.53)$ & $2.0 \times 10^{-5}$ \\
\hline Biliary tract & 387 & $0.67(0.34,1.32)$ & 0.25 & $1.06(0.65,1.72)$ & 0.81 & $1.33(0.31,5.63)$ & 0.70 & $0.77(0.42,1.43)$ & 0.41 \\
\hline Sex-specific & & & & & & & & & \\
\hline Breast (UKBB) & 13,666 & $1.10(1.00,1.22)$ & 0.05 & $1.06(0.98,1.16)$ & 0.16 & $1.18(0.89,1.56)$ & 0.25 & $0.94(0.81,1.10)$ & 0.47 \\
\hline Breast (BCAC) & 122,977 & $0.99(0.94,1.03)$ & 0.62 & $0.99(0.96,1.03)$ & 0.65 & $0.98(0.88,1.08)$ & 0.66 & $1.01(0.96,1.06)$ & 0.75 \\
\hline Breast ER+ (BCAC) & 69,501 & $1.01(0.96,1.07)$ & 0.73 & $1.00(0.97,1.03)$ & 0.85 & $1.03(0.91,1.17)$ & 0.63 & $0.98(0.93,1.05)$ & 0.60 \\
\hline Breast ER- (BCAC) & 21,468 & $0.93(0.85,1.01)$ & 0.07 & $0.95(0.89,1.01)$ & 0.08 & $0.85(0.70,1.02)$ & 0.09 & $1.07(0.98,1.18)$ & 0.14 \\
\hline Uterus & 1931 & $0.99(0.79,1.24)$ & 0.95 & $0.98(0.84,1.15)$ & 0.83 & $0.94(0.58,1.53)$ & 0.80 & $1.06(0.83,1.33)$ & 0.65 \\
\hline Cervix & 1928 & $1.11(0.88,1.41)$ & 0.38 & $1.06(0.88,1.27)$ & 0.53 & $1.18(0.67,2.08)$ & 0.57 & $0.97(0.73,1.30)$ & 0.84 \\
\hline Ovary & 1520 & $0.98(0.69,1.39)$ & 0.91 & $0.97(0.76,1.24)$ & 0.82 & $0.92(0.43,1.94)$ & 0.82 & $1.08(0.76,1.53)$ & 0.66 \\
\hline Prostate & 7872 & $1.10(0.91,1.33)$ & 0.34 & $1.07(0.94,1.22)$ & 0.32 & $1.24(0.83,1.85)$ & 0.29 & $0.91(0.74,1.12)$ & 0.40 \\
\hline Testis & 735 & $1.11(0.77,1.60)$ & 0.57 & $1.11(0.86,1.44)$ & 0.43 & $1.42(0.64,3.12)$ & 0.39 & $0.81(0.56,1.19)$ & 0.29 \\
\hline \multicolumn{10}{|l|}{ Urinary tract } \\
\hline Bladder & 2588 & $1.08(0.89,1.31)$ & 0.46 & $1.045(0.91,1.2)$ & 0.51 & $1.15(0.75,1.76)$ & 0.52 & $0.96(0.78,1.17)$ & 0.67 \\
\hline Kidney & 1310 & $0.97(0.73,1.28)$ & 0.82 & $1.01(0.82,1.23)$ & 0.95 & $1.07(0.58,1.96)$ & 0.83 & $0.93(0.70,1.23)$ & 0.60 \\
\hline \multicolumn{10}{|l|}{ Blood/bone marrow/lymph } \\
\hline Leukemia & 1403 & $0.99(0.77,1.29)$ & 0.96 & $0.98(0.81,1.18)$ & 0.82 & $0.91(0.51,1.61)$ & 0.75 & $1.07(0.81,1.40)$ & 0.65 \\
\hline Non-Hodgkin lymphoma & 2296 & $0.92(0.75,1.13)$ & 0.43 & $0.95(0.82,1.10)$ & 0.50 & $0.87(0.55,1.36)$ & 0.53 & $1.05(0.84,1.30)$ & 0.67 \\
\hline $\begin{array}{c}\text { Multiple myeloma } \\
\text { Other }\end{array}$ & 656 & $0.80(0.41,1.59)$ & 0.53 & $0.80(0.60,1.08)$ & 0.15 & $0.51(0.21,1.22)$ & 0.13 & $1.33(0.82,2.14)$ & 0.25 \\
\hline Thyroid & 375 & $1.68(0.63,4.49)$ & 0.30 & $1.54(0.81,2.93)$ & 0.19 & $4.06(0.65,25.53)$ & 0.14 & $0.51(0.21,1.24)$ & 0.14 \\
\hline Lung & 2838 & $0.93(0.77,1.12)$ & 0.43 & $0.93(0.82,1.07)$ & 0.31 & $0.80(0.54,1.20)$ & 0.29 & $1.13(0.94,1.37)$ & 0.20 \\
\hline Melanoma & 4869 & $0.96(0.83,1.11)$ & 0.58 & $0.98(0.88,1.08)$ & 0.66 & $0.94(0.69,1.28)$ & 0.71 & $1.01(0.87,1.18)$ & 0.86 \\
\hline
\end{tabular}

BCAC indicates Breast Cancer Association Consortium; CI; confidence interval; ER, estrogen receptor; OR, odds ratio; UKBB, UK Biobank. All estimations were based on the inverse-variance weighted method with random-effects. 
Table 3. Associations between genetically predicted iron status, based on three primary SNPs, and liver cancer in sensitivity analyses.

\begin{tabular}{|c|c|c|c|c|c|c|c|c|}
\hline \multirow{2}{*}{ SNP (Gene) or Method } & \multicolumn{2}{|c|}{ Serum Iron } & \multicolumn{2}{|c|}{ Transferrin Saturation } & \multicolumn{2}{|c|}{ Ferritin } & \multicolumn{2}{|c|}{ Transferrin } \\
\hline & OR $(95 \%$ CI $)$ & $p$ & OR $(95 \%$ CI $)$ & $p$ & OR (95\% CI) & $p$ & OR $(95 \%$ CI $)$ & $p$ \\
\hline rs1800562 (HFE) & $6.65(2.78,15.9)$ & $2.02 \times 10^{-5}$ & $2.94(1.79,4.82)$ & $2.02 \times 10^{-5}$ & $21.0(5.18,85.4)$ & $2.02 \times 10^{-5}$ & $0.27(0.15,0.50)$ & $2.02 \times 10^{-5}$ \\
\hline rs1799945 (HFE) & $1.01(0.32,3.16)$ & 0.99 & $1.01(0.40,2.56)$ & 0.99 & $1.03(0.04,28.3)$ & 0.99 & $0.98(0.15,6.50)$ & 0.99 \\
\hline rs855791 (TMPRSS6) & $1.54(0.65,3.64)$ & 0.32 & $1.51(0.67,3.42)$ & 0.32 & $4.14(0.24,70.3)$ & 0.32 & $0.17(0.01,5.82)$ & 0.32 \\
\hline IVW-Random effects & $2.45(0.81,7.45)$ & 0.11 & $2.11(1.16,3.83)$ & 0.01 & $10.9(2.44,48.6)$ & $2.00 \times 10^{-3}$ & $0.30(0.17,0.53)$ & $2.99 \times 10^{-5}$ \\
\hline Weighted median & $2.08(0.97,4.47)$ & 0.06 & $2.14(1.39,3.31)$ & $1.00 \times 10^{-3}$ & $12.1(3.24,45.2)$ & $3.43 \times 10^{-4}$ & $0.30(0.17,0.53)$ & $3.31 \times 10^{-5}$ \\
\hline MR-Egger & $49.0(5.64,424)$ & $4.17 \times 10^{-4}$ & $4.36(1.87,10.1)$ & $1.00 \times 10^{-3}$ & $46.4(5.47,394)$ & $4.38 \times 10^{-4}$ & $0.28(0.11,0.70)$ & $7.00 \times 10^{-3}$ \\
\hline Heterogeneity $\left(\mathrm{I}^{2}\right)$ & $76(23,93)$ & 0.01 & $58(0,88)$ & 0.09 & $38(0,81)$ & 0.20 & $0(0,90)$ & 0.43 \\
\hline Pleiotropy (Intercept) & NA & $5.00 \times 10^{-3}$ & NA & 0.06 & NA & 0.11 & NA & 0.76 \\
\hline
\end{tabular}

CI indicates confidence interval; IVW, inverse-variance weighted; NA, Not Available; OR, odds ratio; SNP, single nucleotide polymorphism.

Table 4. Associations between genetically predicted iron status, based on 3 primary SNPs, and brain cancer in sensitivity analyses.

\begin{tabular}{|c|c|c|c|c|c|c|c|c|}
\hline \multirow{2}{*}{ SNP (Gene) or Method } & \multicolumn{2}{|c|}{ Serum Iron } & \multicolumn{2}{|c|}{ Transferrin Saturation } & \multicolumn{2}{|c|}{ Ferritin } & \multicolumn{2}{|c|}{ Transferrin } \\
\hline & OR $(95 \% \mathrm{CI})$ & $p$ & OR $(95 \% \mathrm{CI})$ & $p$ & OR $(95 \% \mathrm{CI})$ & $p$ & OR $(95 \% \mathrm{CI})$ & $p$ \\
\hline rs1800562 (HFE) & $1.09(0.53,2.24)$ & 0.82 & $1.07(0.59,1.93)$ & 0.82 & $1.28(0.16,10.4)$ & 0.82 & $5.04(0.54,47.4)$ & 0.82 \\
\hline rs1799945 (HFE) & $0.54(0.31,0.95)$ & 0.16 & $0.69(0.41,1.16)$ & 0.16 & $0.27(0.05,1.65)$ & 0.16 & $0.87(0.26,2.88)$ & 0.16 \\
\hline rs855791 (TMPRSS6) & $0.67(0.39,1.16)$ & 0.03 & $0.71(0.51,0.97)$ & 0.03 & $0.37(0.15,0.92)$ & 0.03 & $1.52(1.04,2.23)$ & 0.03 \\
\hline IVW-Random effects & $0.69(0.48,1.00)$ & 0.05 & $0.75(0.59,0.97)$ & 0.03 & $0.41(0.20,0.88)$ & 0.02 & $1.49(1.04,2.14)$ & 0.03 \\
\hline Weighted median & $0.65(0.44,0.96)$ & 0.03 & $0.71(0.53,0.93)$ & 0.02 & $0.37(0.16,0.83)$ & 0.02 & $1.46(1.01,2.13)$ & 0.05 \\
\hline MR-Egger & $0.35(0.07,1.63)$ & 0.18 & $0.68(0.36,1.29)$ & 0.24 & $0.36(0.08,1.69)$ & 0.19 & $1.38(0.74,2.57)$ & 0.31 \\
\hline Heterogeneity $\left(\mathrm{I}^{2}\right)$ & $11(0,91)$ & 0.33 & $0(0,90)$ & 0.44 & $0(0,90)$ & 0.51 & $0(0,90)$ & 0.38 \\
\hline Pleiotropy (Intercept) & NA & 0.37 & NA & 0.72 & NA & 0.82 & NA & 0.72 \\
\hline
\end{tabular}

CI indicates confidence interval; IVW, inverse-variance weighted; NA, Not Available; OR, odds ratio; SNP, single nucleotide polymorphism. 
In the sensitivity analyses using all SNPs for each iron status biomarker, the direction and magnitude of the effects of iron status on liver and brain cancer remained, but only the associations of serum iron levels with brain cancer and of transferrin saturation with liver and brain cancer reached the conventional level of significance (Supplementary Figures S2-S5). The associations did not remain after removing rs1800562 (Supplementary Table S2).

The three SNPs used as instrumental variables for the main analysis were associated with blood cells, $\mathrm{HbA} 1 \mathrm{c}$ and blood pressure at genome-wide significance. Rs1800562 in HFE was additionally related to some other traits, such as height, lipids and pulse rate (Supplementary Table S3). The other SNPs included in the secondary analysis had pleiotropic associations with multiple traits, such as blood cells, cardiovascular diseases, blood lipids, plasma fatty acids, interleukins, pulse rate, asthma and heart rate.

\section{Discussion}

The present two-sample MR study is the first to systematically evaluate the causal role of iron status for a wide range of cancers. It showed that genetically high iron status was associated with higher risk of liver cancer and lower risk of brain cancer. These associations were driven by rs 1800562 , which had the largest impact on iron status among the instrumental variables. There was no evidence in support of a causal association of high iron status with overall cancer and 20 other cancers.

Our results are in line with abundant data from observational and experimental studies, indicating carcinogenic effects of high iron levels on liver cancer. In a recent meta-analysis of cohort and case-control studies, serum ferritin (6 studies) and serum iron (3 studies) levels in the highest category were associated with 1.5- and 2.5-fold increases in risk of liver cancer, respectively, when compared to levels in the lowest group [9]. Moreover, high transferrin saturation ( $\geq 60 \%$ versus $<50 \%)$ was reported to be associated with a significant 5.9-fold higher risk of liver cancer in a prospective cohort of 8763 Danish adults followed up for 15 years [24]. In addition, hepatocellular carcinoma risk is substantially increased among individuals with hemochromatosis gene mutations and in hemochromatosis patients diagnosed by elevated serum transferrin saturation and ferritin levels $[1,25,26]$. Notably, the two HFE SNPs included in this study are known to cause forms of haemochromatosis, with rs1800562 (C282Y) causing a particularly serious type, and rs1799945 (H63D) causing a milder form $[27,28]$. These SNPs are related to decreased levels of hepcidin, thereby causing the increase of iron absorption and parenchymal deposition (iron overload) in the liver [29]. Our findings are therefore consistent with previous reports that heterozygosity and combined heterozygosity of these two SNPs are associated with liver cancer. A recent review indicated that iron overload was a common consequence of an excessive and prolonged consumption of alcohol. An increased level of iron was observed in both serum and within cells, hepatocytes in particular [30]. Furthermore, a dose-response relationship has been observed between alcohol consumption and liver cancer [31]. In the present study, SNPs used for iron status were not related to alcohol consumption, implying that the observed association between iron status and liver cancer is unlikely driven by alcohol consumption. Thus, it is speculated that elevated iron status may partly mediate the pathway from alcohol consumption to liver cancer, which is in line with our findings indirectly.

These epidemiologic associations between iron status and liver cancer have been further supported by animal studies. Long-Evans Cinnamon rats fed an iron-deficient diet for 65 weeks exhibit lower risk of fulminant hepatitis, hepatic fibrosis, and subsequent hepatocarcinogenesis compared with those fed a normal diet after a 65-week intervention [32]. Similarly, iron deposition in the liver is likely to promote liver cancer in humans as patients with hepatocellular carcinoma complicating noncirrhotic liver [33] and non-alcoholic steato-hepatitis [34] have higher iron deposition in non-tumorous areas of the liver than those without cancer. A range of putative carcinomic mechanisms of high iron status in the liver have been proposed, including deoxyribonucleic acid damage and cytotoxic by-products of lipid peroxidation caused by reactive oxygen species, free radicals, ferroptosis $[26,35,36]$, facilitation of cancer growth [37], and lymphocyte and macrophage functions impairment [35]. 
This MR study, therefore, adds strong causal evidence to previous observational and experimental studies that high physiological levels of iron status promote liver cancer. This has important clinical implications with regards to the treatment of borderline anaemia and the continuation of iron therapies in anaemic patients after their iron status has been corrected, particularly if patients are asymptomatic. Conversely, there may be a benefit to careful reduction of iron indices for reducing liver cancer risk in patients with high physiological iron status or in patients who are at risk due to other factors such as alcohol-related liver disease or viral hepatitis. Further studies should address the risk of liver cancer in patients receiving long-term iron therapy with borderline anaemia or normalized iron status. Similarly, the benefits of regular phlebotomy or iron chelation seen with hemochromatosis may potentially be extended in specific groups of patients with high physiological iron levels.

Data on the association between iron status and brain cancer are limited. Nevertheless, iron-related gene expression has been reported to be dysregulated in brain tumours, such as elevated HFE expression in meningioma [38] an iron redox state affects brain cancer severity. These studies suggest that iron homeostasis may be involved in the development of brain cancer, but more broadly, several studies have shown that iron has important effects on the brain. Iron deficiency causes neuropsychologic impairment [39] and behavioural dysfunction [40], and even contributed to long-term organic change in brain, such as dopamine metabolism, myelination, and hippocampal structure and function [40]. Additionally, it has been postulated that both iron excess and deficiency lead to oxidative deoxyribonucleic acid damage, thereby increasing the risk of cancer [3]. Consistent with a protective effect of low iron levels on brain cancer risk, iron chelation with deferoxamine has been suggested as a novel approach to therapy for brain cancer, due to antiproliferative effects, which are mediated by an intracellular pool of iron [37]. Genetic evidence also lends support to our findings stating that the TMPRSS6 gene exerts an important role in the absorption of dietary iron via intestines and transporting out iron from storage sites (particularly in the liver and spleen) to other organs via the bloodstream [41]. Iron status in the brain can be different from other organs due to the presence of the blood-brain barrier. It has been well acknowledged that iron is taken up by means of receptor-mediated uptake of iron-transferrin at the blood-brain barrier, thereby transporting iron from the circulation into the brain extracellular space [42]. Our study showed that both lower serum iron and higher transferrin levels were associated with higher risk of brain cancer. An animal study indicated that iron supplementation prevented lead-induced disruption of the blood-brain barrier in the rat development [43]. Population-based studies have also found that iron deficiency caused alternations of brain development and functioning, possibly also affecting the brain barrier [44]. Blood-brain barrier breakdown or alterations in transport systems have been shown to play a vital role in the pathogenesis of many central nervous system diseases, including tumours [45]. Considering the important role of the blood-brain barrier for iron homeostasis and tumours in the brain along with limited and inconsistent findings of the effects of physiologically high iron levels on blood-brain barrier, additional research on the effects of iron status on blood-brain barrier function and on brain cancer risk is warranted. Although our MR study provides causal evidence of an inverse relationship between iron status and overall brain cancer, considering the small number of brain cancer cases, additional studies with larger sample sizes are warranted to verify our findings. Furthermore, the effects of iron on brain cancer types need to be explored.

A consistent, albeit nonsignificant pattern of high iron status in a high risk of thyroid cancer was observed in the present study. In a study with 102 metastatic thyroid cancer patients, serum ferritin levels were higher in the thyroid cancer group than among primary hypothyroid patients [46]. In addition, a recent cell study found a new pathway of E4BP4/G9a/SOSTDC1/hepcidin linking cellular iron dysfunction to thyroid cancer [47]. The potential role of iron status in the development of thyroid cancer needs further investigation.

Findings of observational studies of the association of iron status with other cancers are conflicting or limited $[8,10]$, but particular attention has related to dietary iron intake and colorectal cancer. A systematic review and meta-analysis found that heme iron intake was positively associated with 
risk of colorectal and colon cancer, whereas serum iron levels were inversely associated with these cancers [8]. However, the inverse association with serum iron levels may be related to reverse causality as iron deficiency is a common presentation of colon cancer patients [48]. A prospective cohort study of 35,121 US men found no association between frequent blood donations, which may reduce body iron stores, and colorectal cancer incidence or mortality [49]. Our results are, therefore, in agreement with previous observational evidence, which do not support an important role of iron stores in colorectal carcinogenesis. The previously observed findings for heme iron, which is found in meat, poultry, and seafood, may be related to a local adverse effect of heme iron in the colorectum, such as a promoting effect on intestinal N-nitroso compound formation [50]. Another recent meta-analysis found that heme iron intake (based on six studies) and serum iron levels (based on four studies), but no other iron status biomarkers, were significantly positively associated with risk of breast cancer [10]. The present study showed no evidence supporting a causal association between iron status and breast cancer risk. The discrepancy in findings from this MR study and previous observational studies may be attributed to residual confounding from other compounds in meat, unhealthy dietary patterns or other lifestyle behaviours. No consistent association has been reported between iron status and other cancers, but epidemiological data are scarce [8].

A major strength of this study is the MR study design, which minimizes the confounding and reverse causality seen in observational studies. In addition, we systematically assessed the associations between four individual iron status indicators and 22 cancers using summary-level data from large-scale genetic consortia and cohorts. The studies only used data from European populations. This reduced population stratification bias but confined the transferability of our findings to other populations. A major limitation of this study is that the statistical power was low in several analyses due to a small number of cases for most cancer sites. In addition, this MR study investigated the association of iron status within the normal range with cancer risk. Therefore, our results cannot be used to make inferences regarding the effect of abnormally high serum iron levels caused by, for example, intravenous, long-term oral iron supplementation or hemochromatosis.

\section{Conclusions}

The present study showed that genetically predicted iron status was positively associated with risk of liver cancer and inversely associated with risk of brain cancer but not associated with overall cancer and a number of other site-specific cancers among European-descent individuals. These findings need to be interpreted with caution in light of the influential effect of a single variant in the HFE gene and the limited number of liver and brain cancer cases. Nevertheless, our results, along with observational data, highlight the importance of further research on the impact of adjustment of iron levels within the physiological range, through supplementation or chelation, on risk of these cancers types. In particular, careful consideration may be needed with regards to ongoing supplementation in anaemic patients or those at high risk of liver cancer.

Supplementary Materials: The following are available online at http://www.mdpi.com/2072-6643/12/2/526/s1, Figure S1: Power estimation based on phenotypic variance explained and case number, Figure S2: Association between genetically predicted serum iron levels and cancer using all SNPs $(n=5)$, Figure S3: Association between genetically predicted transferrin saturation and cancer using all SNPs $(n=5)$, Figure S4, Association between genetically predicted $\log 10$ ferritin and cancer using all SNPs $(n=6)$, Figure S5, Association between genetically predicted transferrin and cancer using all SNPs $(n=8)$, Table S1: Information of included studies and consortia, Table S2, Associations of iron status with liver and brain cancer excluding rs1800562, Table S3, Associations of the instrumental variables for iron status with other traits at the genome-wide significance level.

Author Contributions: S.Y. analyzed and interpreted data and wrote the manuscript. P.C., M.V., S.K. and E.G. reviewed the manuscript. A.M.M. and S.B. prepared the data and reviewed the manuscript. S.C.L. designed the research, analyzed and interpreted the data and reviewed the manuscript. All authors read and approved the final manuscript.

Funding: Funding for this study came from the Swedish Research Council for Health, Working Life and Welfare (Forte) and the Swedish Research Council (Vetenskapsrådet). Siddhartha Kar is supported by a Cancer Research UK programme grant, the Integrative Cancer Epidemiology Programme (C18281/A19169), and a Junior Research 
Fellowship from Homerton College, Cambridge. Stephen Burgess is supported by a Sir Henry Dale Fellowship, jointly funded by the Wellcome Trust and the Royal Society (Grant Number 204623/Z/16/Z).

Acknowledgments: Summary-level data for genetic associations with the cancers have been contributed by the Breast Cancer Association Consortium and UK Biobank. The authors thank all investigators for sharing these data. The analyses of UK Biobank data were conducted under application 29202.

Conflicts of Interest: The authors declare no conflict of interest.

\section{References}

1. Fracanzani, A.L.; Conte, D.; Fraquelli, M.; Taioli, E.; Mattioli, M.; Losco, A.; Fargion, S. Increased cancer risk in a cohort of 230 patients with hereditary hemochromatosis in comparison to matched control patients with non-iron-related chronic liver disease. Hepatology 2001, 33, 647-651. [CrossRef] [PubMed]

2. Elmberg, M.; Hultcrantz, R.; Ekbom, A.; Brandt, L.; Olsson, S.; Olsson, R.; Lindgren, S.; Lööf, L.; Stål, P.; Wallerstedt, S.; et al. Cancer risk in patients with hereditary hemochromatosis and in their first-degree relatives. Gastroenterology 2003, 125, 1733-1741. [CrossRef] [PubMed]

3. Torti, S.V.; Torti, F.M. Iron and cancer: More ore to be mined. Nat. Rev. Cancer 2013, 13, 342-355. [CrossRef] [PubMed]

4. Zacharski, L.R.; Chow, B.K.; Howes, P.S.; Shamayeva, G.; Baron, J.A.; Dalman, R.L.; Malenka, D.J.; Ozaki, C.K.; Lavori, P.W. Decreased cancer risk after iron reduction in patients with peripheral arterial disease: Results from a randomized trial. J. Natl. Cancer Inst. 2008, 100, 996-1002. [CrossRef] [PubMed]

5. Osborne, N.J.; Gurrin, L.C.; Allen, K.J.; Constantine, C.C.; Delatycki, M.B.; McLaren, C.E.; Gertig, D.M.; Anderson, G.J.; Southey, M.C.; Olynyk, J.K.; et al. HFE C282Y homozygotes are at increased risk of breast and colorectal cancer. Hepatology 2010, 51, 1311-1318. [CrossRef] [PubMed]

6. Fargion, S.; Valenti, L.; Fracanzani, A.L. Hemochromatosis gene (HFE) mutations and cancer risk: Expanding the clinical manifestations of hereditary iron overload. Hepatology 2010, 51, 1119-1121. [CrossRef]

7. Corley, D.A.; Kubo, A.; Levin, T.R.; Habel, L.; Zhao, W.; Leighton, P.; Rumore, G.; Quesenberry, C.; Buffler, P.; Block, G. Iron intake and body iron stores as risk factors for Barrett's esophagus: A community-based study. Am. J. Gastroenterol. 2008, 103, 2997-3004. [CrossRef]

8. Fonseca-Nunes, A.; Jakszyn, P.; Agudo, A. Iron and cancer risk-A systematic review and meta-analysis of the epidemiological evidence. Cancer Epidemiol. Biomark. Prev. 2014, 23, 12-31. [CrossRef]

9. Tran, K.T.; Coleman, H.G.; McCain, R.S.; Cardwell, C.R. Serum Biomarkers of Iron Status and Risk of Primary Liver Cancer: A Systematic Review and Meta-Analysis. Nutr. Cancer 2019, 71, 1365-1373. [CrossRef]

10. Chang, V.C.; Cotterchio, M.; Khoo, E. Iron intake; body iron status; and risk of breast cancer: A systematic review and meta-analysis. BMC Cancer 2019, 19, 543. [CrossRef]

11. Chen, H.F.; Wu, L.X.; Li, X.F.; Zhu, Y.C.; Wang, W.X.; Xu, C.W.; Xie, D.F.; Huang, J.H.; Du, K.Q. A meta-analysis of association between serum iron levels and lung cancer risk. Cell Mol. Biol. 2018, 64, 33-37. [CrossRef]

12. Smith, G.D.; Ebrahim, S. 'Mendelian randomization': Can genetic epidemiology contribute to understanding environmental determinants of disease? Int. J. Epidemiol. 2003, 32, 1-22. [CrossRef] [PubMed]

13. Benyamin, B.; Esko, T.; Ried, J.S.; Radhakrishnan, A.; Vermeulen, S.H.; Traglia, M.; Anderson, D.; Broer, L.; Podmore, C.; Luan, J.; et al. Novel loci affecting iron homeostasis and their effects in individuals at risk for hemochromatosis. Nat. Commun. 2014, 5, 4926. [CrossRef] [PubMed]

14. Gill, D.; Del Greco, M.F.; Walker, A.P.; Srai, S.K.S.; Laffan, M.A.; Minelli, C. The Effect of Iron Status on Risk of Coronary Artery Disease: A Mendelian Randomization Study-Brief Report. Arterioscler. Thromb. Vasc. Biol. 2017, 37, 1788-1792. [CrossRef] [PubMed]

15. Gill, D.; Monori, G.; Tzoulaki, I.; Dehghan, A. Iron Status and Risk of Stroke. Stroke 2018, 49, $2815-2821$. [CrossRef] [PubMed]

16. Gill, D.; Brewer, C.F.; Monori, G.; Tregouet, D.A.; Franceschini, N.; Giambartolomei, C.; INVENT, C.; Tzoulaki, I.; Dehghan, A. Effects of Genetically Determined Iron Status on Risk of Venous Thromboembolism and Carotid Atherosclerotic Disease: A Mendelian Randomization Study. J. Am. Heart Assoc. 2019, 8, e012994. [CrossRef]

17. Michailidou, K.; Lindstrom, S.; Dennis, J.; Beesley, J.; Hui, S.; Kar, S.; Lemaçon, A.; Soucy, P.; Glubb, D.; Rostamianfar, A.; et al. Association analysis identifies 65 new breast cancer risk loci. Nature 2017, 551, 92-94. [CrossRef] 
18. Sudlow, C.; Gallacher, J.; Allen, N.; Beral, V.; Burton, P.; Danesh, J.; Downey, P.; Elliott, P.; Green, J.; Landray, M.; et al. UK biobank: An open access resource for identifying the causes of a wide range of complex diseases of middle and old age. PLoS Med. 2015, 12, e1001779. [CrossRef]

19. Bowden, J.; Davey Smith, G.; Haycock, P.C.; Burgess, S. Consistent Estimation in Mendelian Randomization with Some Invalid Instruments Using a Weighted Median Estimator. Genet. Epidemiol. 2016, 40, 304-314. [CrossRef]

20. Burgess, S.; Thompson, S.G. Interpreting findings from Mendelian randomization using the MR-Egger method. Eur. J. Epidemiol. 2017, 32, 377-389. [CrossRef]

21. Burgess, S. Sample size and power calculations in Mendelian randomization with a single instrumental variable and a binary outcome. Int. J. Epidemiol. 2014, 43, 922-929. [CrossRef] [PubMed]

22. Kamat, M.A.; Blackshaw, J.A.; Young, R.; Surendran, P.; Burgess, S.; Danesh, J.; Butterworth, A.S.; Staley, J.R. PhenoScanner V2: An expanded tool for searching human genotype-phenotype associations. Bioinformatics 2019, 35, 4851-4853. [CrossRef] [PubMed]

23. Sterne, J.A.; Davey Smith, G. Sifting the evidence-what's wrong with significance tests? Phys. Ther. 2001, 322, 226-231. [CrossRef] [PubMed]

24. Ellervik, C.; Tybjaerg-Hansen, A.; Nordestgaard, B.G. Risk of cancer by transferrin saturation levels and haemochromatosis genotype: Population-based study and meta-analysis. J. Intern. Med. 2012, 271, 51-63. [CrossRef]

25. Nahon, P.; Sutton, A.; Rufat, P.; Ziol, M.; Thabut, G.; Schischmanoff, P.O.; Vidaud, D.; Charnaux, N.; Couvert, P.; Ganne-Carrie, N.; et al. Liver iron; HFE gene mutations; and hepatocellular carcinoma occurrence in patients with cirrhosis. Gastroenterology 2008, 134, 102-110. [CrossRef]

26. Kowdley, K.V. Iron; hemochromatosis; and hepatocellular carcinoma. Gastroenterology 2004, 127, S79-S86. [CrossRef]

27. Ye, Q.; Qian, B.X.; Yin, W.L.; Wang, F.M.; Han, T. Association between the HFE C282Y; H63D Polymorphisms and the Risks of Non-Alcoholic Fatty Liver Disease; Liver Cirrhosis and Hepatocellular Carcinoma: An Updated Systematic Review and Meta-Analysis of 5758 Cases and 14741 Controls. PLoS ONE 2016, 11, e0163423. [CrossRef]

28. Hemochromatosis-SNPedia. Available online: https://www.snpedia.com/index.php/Hemochromatosis (accessed on 1 November 2019).

29. Valenti, L.; Fracanzani, A.L.; Bugianesi, E.; Dongiovanni, P.; Galmozzi, E.; Vanni, E.; Canavesi, E.; Lattuada, E.; Roviaro, G.; Marchesini, G.; et al. HFE genotype; parenchymal iron accumulation; and liver fibrosis in patients with nonalcoholic fatty liver disease. Gastroenterology 2010, 138, 905-912. [CrossRef]

30. Grochowski, C.; Blicharska, E.; Baj, J.; Mierzwińska, A.; Brzozowska, K.; Forma, A.; Maciejewski, R. Serum iron, Magnesium, Copper, and Manganese Levels in Alcoholism: A Systematic Review. Molecules 2019, 24, 1361. [CrossRef]

31. Turati, F.; Galeone, C.; Rota, M.; Pelucchi, C.; Negri, E.; Bagnardi, V.; Corrao, G.; Boffetta, P.; La Vecchia, C. Alcohol and liver cancer: A systematic review and meta-analysis of prospective studies. Ann. Oncol. 2014, 25, 1526-1535. [CrossRef]

32. Kato, J.; Kobune, M.; Kohgo, Y.; Sugawara, N.; Hisai, H.; Nakamura, T.; Sakamaki, S.; Sawada, N.; Niitsu, Y. Hepatic iron deprivation prevents spontaneous development of fulminant hepatitis and liver cancer in Long-Evans Cinnamon rats. J. Clin. Investig. 1996, 98, 923-929. [CrossRef] [PubMed]

33. Turlin, B.; Juguet, F.; Moirand, R.; Le Quilleuc, D.; Loreal, O.; Campion, J.P.; Launois, B.; Ramée, M.P.; Brissot, P.; Deugnier, Y. Increased liver iron stores in patients with hepatocellular carcinoma developed on a noncirrhotic liver. Hepatology 1995, 22, 446-450. [PubMed]

34. Sorrentino, P.; D’Angelo, S.; Ferbo, U.; Micheli, P.; Bracigliano, A.; Vecchione, R. Liver iron excess in patients with hepatocellular carcinoma developed on non-alcoholic steato-hepatitis. J. Hepatol. 2009, 50, 351-357. [CrossRef] [PubMed]

35. Deugnier, Y.; Turlin, B. Iron and hepatocellular carcinoma. J. Gastroenterol. Hepatol. 2011, 16, $491-494$. [CrossRef]

36. Kew, M.C. Hepatic iron overload and hepatocellular carcinoma. Cancer Lett. 2009, 286, 38-43. [CrossRef]

37. Dayani, P.N.; Bishop, M.C.; Black, K.; Zeltzer, P.M. Desferoxamine (DFO)—Mediated iron chelation: Rationale for a novel approach to therapy for brain cancer. J. Neurooncol. 2004, 67, 367-377. [CrossRef] 
38. Hanninen, M.M.; Haapasalo, J.; Haapasalo, H.; Fleming, R.E.; Britton, R.S.; Bacon, B.R.; Parkkila, S. Expression of iron-related genes in human brain and brain tumors. BMC Neurosci. 2009, 10, 36. [CrossRef]

39. Sandstead, H.H. Causes of iron and zinc deficiencies and their effects on brain. J. Nutr. 2000, 130, 347S-349S. [CrossRef]

40. Georgieff, M.K. Long-term brain and behavioral consequences of early iron deficiency. Nutr. Rev. 2011, 69, S43-S48. [CrossRef]

41. Reference, G.H. TMPRSS6 Gene. Available online: https://www.ncbi.nlm.nih.gov/pubmed/ (accessed on 5 November 2019).

42. Moos, T. Brain iron homeostasis. Dan. Med. Bull. 2002, 49, 279-301.

43. Wang, Q.; Luo, W.; Zheng, W.; Liu, Y.; Xu, H.; Zheng, G.; Dai, Z.; Zhang, W.; Chen, Y.; Chen, J. Iron supplement prevents lead-induced disruption of the blood-brain barrier during rat development. Toxicol. Appl. Pharmacol. 2007, 219, 33-41. [CrossRef] [PubMed]

44. Cusick, S.E.; Georgieff, M.K.; Rao, R. Approaches for Reducing the Risk of Early-Life Iron Deficiency-Induced Brain Dysfunction in Children. Nutrients 2018, 10, 227. [CrossRef] [PubMed]

45. Persidsky, Y.; Ramirez, S.H.; Haorah, J.; Kanmogne, G.D. Blood-brain barrier: Structural components and function under physiologic and pathologic conditions. J. Neuroimmune Pharmacol. 2006, 1, 223-236. [CrossRef]

46. Deshpande, U.R.; Nadkarni, G.D.; Samuel, A.M. Serum ferritin in thyroid cancer. Thyroid 1993, 3, 301-303. [CrossRef] [PubMed]

47. Zhou, Q.; Chen, J.; Feng, J.; Wang, J. E4BP4 promotes thyroid cancer proliferation by modulating iron homeostasis through repression of hepcidin. Cell Death Dis. 2018, 9, 987. [CrossRef]

48. Wilson, M.J.; Dekker, J.W.T.; Harlaar, J.J.; Jeekel, J.; Schipperus, M.; Zwaginga, J.J. The role of preoperative iron deficiency in colorectal cancer patients: Prevalence and treatment. Int. J. Colorectal Dis. 2017, 32, 1617-1624. [CrossRef]

49. Zhang, X.; Ma, J.; Wu, K.; Chan, A.T.; Fuchs, C.S.; Giovannucci, E.L. Blood donation and colorectal cancer incidence and mortality in men. PLoS ONE 2012, 7, e39319. [CrossRef]

50. Cross, A.J.; Pollock, J.R.; Bingham, S.A. Haem, not protein or inorganic iron, is responsible for endogenous intestinal N-nitrosation arising from red meat. Cancer Res. 2003, 63, 2358-2360. 